\title{
Chapter 25 \\ Life Cycle Management as a Way \\ to Operationalize the Creating Shared \\ Value Concept in the Food and Beverage \\ Industry: A Case Study
}

\author{
Angela Adams, Urs Schenker, and Yves Loerincik
}

\begin{abstract}
Nestlé is using life cycle management approaches to operationalize the Creating Shared Value concept that is strongly promoted by the company. Following the success of the implementation of PIQET, an LCA software for packaging designers to integrate environmental criteria in their decision-making, Nestlé has decided to develop a specific tool moving from packaging to product ecodesign tool. This tool, EcodEX, is linked to the recipe system of the company to facilitate the realization and improve the quality of LCA. The number of users has increased to reach 700 people today. However, there have been many challenges to overcome, such as: the availability and the management of inventory data, following-up the latest methodology, as well as the training and education of users.
\end{abstract}

Keywords Creating Shared Value - Data development - Data management • Ecodesign $\bullet$ EcodEX $\bullet$ Education $\bullet$ Life cycle assessment $\bullet$ Life cycle management - Nestlé • PIQET • Training

\section{Introduction}

Systematically applying Creating Shared Value principles in practice and integrating them into the way a company is doing business is a huge challenge, and in some cases, even a paradigm change. Life cycle management is an important and powerful approach that Nestlé uses to operationalize creating shared value.

\footnotetext{
A. Adams $(\triangle) \bullet Y$. Loerincik

Quantis-International, EPFL Innovation Park, Bât D, CH-1015 Lausanne, Switzerland

e-mail: angela.adams@quantis-intl.com

U. Schenker

Nestlé Research Center, Vers-chez-les-Blanc, CH-1000 Lausanne 26, Switzerland
} 
Nestlé adopted a simplified ecodesign approach early in the design phase since many years through the implementation of PIQET (Karli et al. 2010), a tool that optimizes the environmental performance of packaging. Since then, packaging designers using life cycle assessment to support their design choices is significantly increased.

Several studies (Humbert et al. 2009; Kim et al. 2013) have shown that in the food and beverage industry the product itself generally dominates the impacts of any life cycle assessment (LCA) and that, comparatively, packaging has a limited impact on the environment. The resolution to expand the ecodesign approach to the products was therefore an obvious decision for Nestlé. To do so, a new simplified ecodesign tool, EcodEX (Schenker et al. 2014), has been adopted to evaluate the current and new products, taking into account the whole life cycle, including consumer behavior.

\section{Implementing EcodEX: A Product Design Tool at Nestlé}

\subsection{Initial Brief}

Prior to the choice to develop a specific tool for product ecodesign, a detailed requirement analysis was completed indicating that the ideal tool has to:

- Be user-friendly: because the users are not LCA experts and results must be quickly generated at a moment's notice, the tool has to be easy and quickly ready for use.

- Be linked to Nestlé's recipe management IT system in order to ensure high quality ingredient data, processing data and eventually other types of data, without spending a lot of time on data collection.

- Give reliable results: although the tool is a simplified tool, the results must be as robust as possible to support relevant decisions.

The direct benefits of implementing EcodEX include products with better environmental performances as well as conscious decision-making where a design alternative with a better environmental performance is selected (e.g., for reasons other than environmental reasons, such as legal compliance, consumer preference or quality).

\subsection{A Growing Number of Users}

EcodEX (Selerant Corporation 2015) has been regularly used by the Nestlé R\&D community for approximately 1 year now. To date, approximately 700 users have analyzed over 900 LCA scenarios. In addition, approximately 1,5000 packaging scenarios which were generated using PIQET over the last 6 years are available. The 
results of these scenarios have influenced the development process of new products in several ways:

A. If the environmental performance of the new design is of less quality than before:

- Product designers may work on an alternative design that improves the environmental performance of the product. In the past, in the absence of EcodEX, designers were not always aware that their designs had negative impacts on the environment.

- The product development may be interrupted or stopped altogether because environmental performance is of key importance to the business unit and market. Without the information provided by EcodEX, decision-makers are not in the position to take timely decisions in the earlier stages of the development process. In cases where LCA studies were performed by an external consultant, the conclusions could not be implemented in the design process.

- A decision may be made where the project goes ahead despite the adverse environmental effects because of other criteria such as consumer preference, nutrition, quality, or legal requirements. In this case, EcodEX provided all information available for the decision making process and may lead to follow-up projects in order to improve.

B. If the environmental performance of the new design improves, the results can be shared internally to gain support for the new product development. This may result in a faster adoption of the product, or the use of the product in other markets. Many EcodEX users consider the tool to be very useful to address upcoming needs for consumers.

The main objective of the ecodesign process is to ensure that the environmental performance of Nestlé's products is taken into account in internal decision-making. An added benefit is that the process identifies external communication opportunities. If in line with the brand positioning, such external communications can be achieved through comprehensive life cycle assessments with external consultants, because EcodEX does not comply directly with the requirements of the ISO 14040/ 14044 standards (ISO 14040 2006) for external communication of comparative assertions, given third party critical reviews are not part of the system. This may be a future development.

\subsubsection{The Tivall Example}

Tivall is a Nestlé brand that offers healthy and nutritious meat substitute products. Tivall products have been specifically developed to be nutritionally balanced and to be a complete source of protein fortified with nutrients that may otherwise be lacking in a meat-free diet. The Tivall range is mainly based on soya and wheat proteins. 
Nestlé has used environmental LCAs to compare the environmental performance of a Tivall vegetarian burger (Hälsans Kök) with a conventional meat burger. The results show that the vegetarian Tivall burgers have a considerably improved environmental performance for all indicators compared to conventional meat burgers. The greenhouse gas emissions, for instance, are approximately $80 \%$ lower. The results show that the vast majority of environmental impacts of the beef burger are related to beef production. For the vegetarian burger, distribution also plays a relatively important role (in addition to the ingredients), because they are distributed while frozen.

The example illustrates that healthy and tasty vegetarian dishes can play an important role in improving the environmental performance of nutrition. Given the evidence for this and other similar studies, Nestlé has launched several R\&D projects to further investigate new sources of protein with improved environmental performance.

\subsubsection{Increasing Internal Knowhow}

Another key benefit of EcodEX is that product designers' understanding of sustainability is greatly increased as they have been trained to do the environmental assessments themselves, rather than picking up the information from LCA reports or being informed about the results and recommendations. At Nestlé, it can very clearly be seen that a systematic and holistic value chain approach is used. This takes into consideration responsible sourcing, ingredients, processing, distribution, consumer use processes, and food waste across the entire cycle, rather than only packaging and transportation distances as was the main focus at the beginning of the sustainability journey of Nestlé.

Life cycle management at Nestlé played an important role in expanding the scope of the eco-design from packaging to the full product life cycle and in the implementation and deployment of EcodEX tool across whole Nestlé. The training has been systematically organized and has a strong influence.

\subsection{Challenges}

The development and implementation of a tool like EcodEX faces many challenges. They can be classified into four main areas: methodology, data, technical, and training. With the exception of technical challenges that will not be addressed in this chapter, hereby we briefly describe the remaining challenges that are very much related to the nature of LCA. 


\subsubsection{Methodology}

The importance of life cycle assessment for external communication is likely to increase in the future. While EcodEX is intended for internal decision-making, consumer communication teams may want to use the results for external communication as well. Therefore, it is important that the methodology in the tool is as closely aligned with externally-developed methodologies as possible. This is not a straightforward task because external methodologies are subject to change and not all requirements are known in sufficient detail. The ISO 14040 sets the frame, and the ENVIFOOD protocol (Food SCP RT 2013) gives additional specifications for the food and beverage sector. With the on-going Product Environmental Footprint (PEF) initiative at the European level (European Commission 2013), a series of Product Category Rules (PCR) will be considered in the tool development. A technical constraint for the tool is therefore able to follow the rapid evolution of the methodology.

\subsubsection{Data}

There are about 10,000 of ingredient specifications in the recipe management system. These specifications had to be matched with a reduced number (couple hundred) available life cycle inventory (LCI) datasets. The need for representative data is critical. Until very recent, the access to good quality LCI profiles was an issue in the food and beverage sector. To address this challenge, Nestlé partnered with external stakeholders to initiate the World Food LCA Database, a multi-company and authority-initiative, led by the consulting firm Quantis, and the research center Agroscope, to mutually develop quality food data (Peano et al. 2012). This project will lead to the development of hundreds of specific LCI profiles in the field of food and beverage, feeding EcodEX, and increasing the accuracy of LCAs. Other database projects such as Agri-footprint have also produced datasets, leaving us with the challenge of data harmonization (methodology, format).

Both the evolution of the methodology and the generation or use of new data leads to two main challenges. The first, which has been briefly discussed above, is to ensure that the tool is capable of following the evolution of methodologies and updating data. The second challenge is to make sure that the process to update and deploy these changes is compatible with the way the tool is used. In particular, an update could have an influence on a decision that is currently being taken or has just been taken by a designer. This therefore implies having a tool to manage data, including quality guidelines or a validation process, for instance, as well as a process to test, update, inform, and support users. Nestlé has developed its own data management guidelines and has a data quality manager. 


\subsubsection{Training and Support}

Last but not least, users have to be extensively trained to gain the necessary understanding on sustainability and life cycle assessment, which inevitably requires time and significant resources. Most users have a background in nutrition, food science, process engineering, or packaging technology, which provides them valuable insight in their focus area. In order to be able to make meaningful decisions on the environmental impacts of the products they develop, they need to expand their skill sets to include this topic. It cannot be expected that the quality of the assessments is comparable to that of a trained LCA specialist at the beginning. Nestlé has trained the designers with the LCP board game, a "serious" game to learn about the concept of life cycle assessment that has proven to be very powerful (Fig. 25.1). But an initial training course is not sufficient. Practice and frequent use of the tool, along with

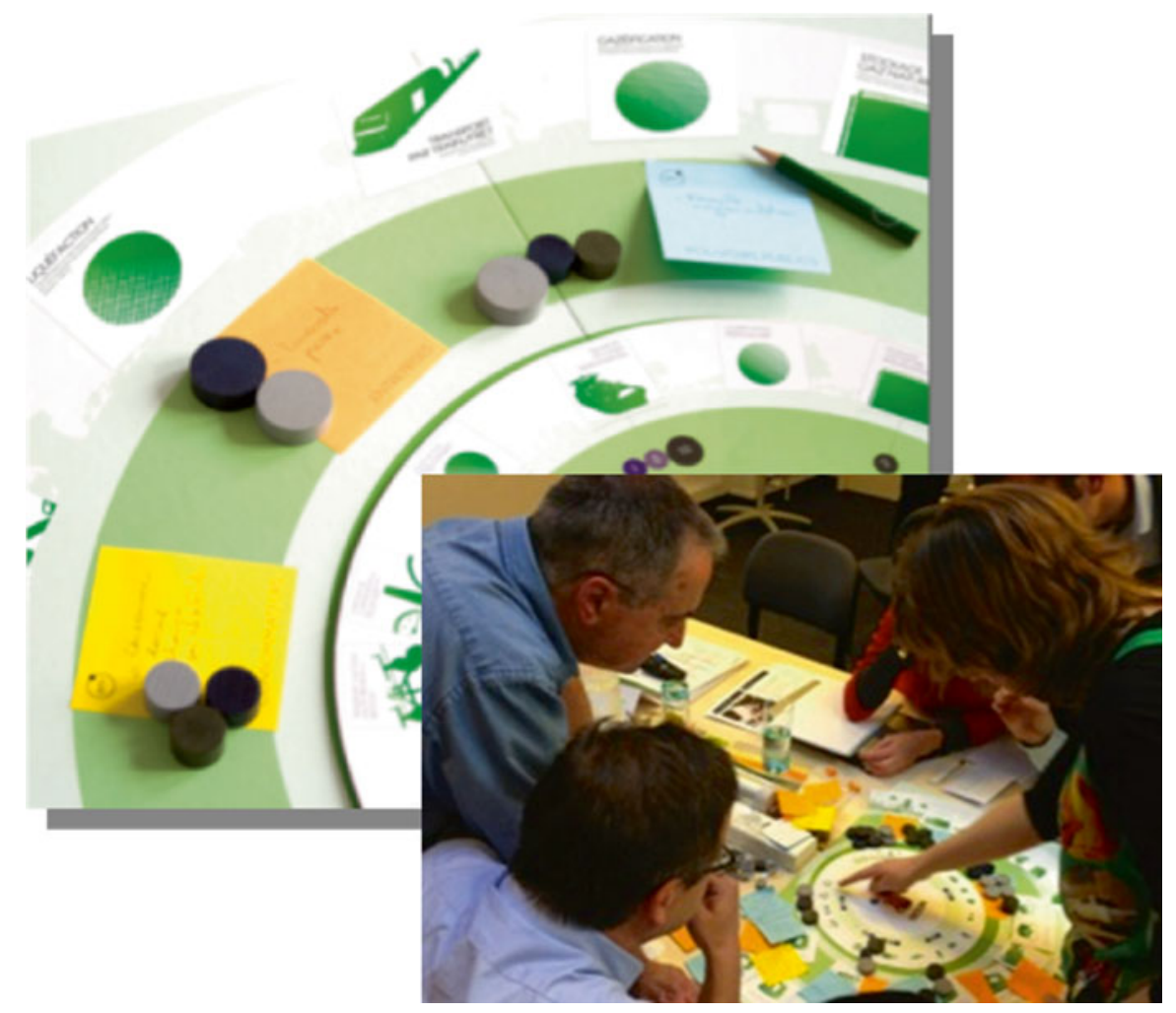

Fig. 25.1 The LCP board game is the ideal tool to educate employees to life cycle thinking and life cycle assessment 
ongoing training through webinars (conference calls with desktop-sharing) and a review of the studies performed ensure that EcodEX users will perform high quality assessments over time.

Another challenge that had to be addressed is the reliability of a simplified ecodesign tool and level of confidence on its outcomes. To verify this, Nestlé decided to evaluate the outcomes of EcodEX against stand-alone LCA case studies.

\section{Conclusion}

Nestlé's objective was to deploy an ecodesign approach for the development of new products. So far it is a success considering the numerous studies that have been performed and the way the tool has influenced designers' decisions accounting for environmental criteria. A technical solution alone, i.e. a tool, is, however, not sufficient to guarantee the success of such a sustainability project. Nestlé's eco-design initiative requires a transversal approach involving diverse teams. Life cycle management ensuring collaboration between different departments within the company and with stakeholders outside the organization is key to implement and sustain such an initiative. Sustainability will certainly be a key differentiator in the coming years, and it is very likely that today's active and engaged companies will have the best tools to be the leaders of tomorrow.

Continuous improvement of Nestlé's environmental performance is challenging and requires a holistic, interdisciplinary, and collaborative approach. A tool like EcodEX combined with appropriate internal business processes provides a very strong base to achieve this.

Open Access This chapter is distributed under the terms of the Creative Commons Attribution Noncommercial License, which permits any noncommercial use, distribution, and reproduction in any medium, provided the original author(s) and source are credited.

\section{References}

European Commission (2013) RECOMMENDATION (2013/179/EU) on the use of common methods to measure and communicate the life cycle environmental performance of products and organisations. Annex II (Product Environmental Footprint (PEF) Guide))

Food SCP RT (2013) ENVIFOOD protocol, environmental assessment of food and drink protocol. European Food Sustainable Consumption and Production Round Table (SCP RT), Working group 1, Brussels

Humbert S, Loerincik Y, Rossi V, Margni M, Jolliet O (2009) Life cycle assessment of spray dried soluble coffee and comparison with alternatives (drip filter and capsule espresso). J Clean Prod 17(15):1351-1358, Environmental Impacts of Food Production and Consumption, A research report completed for the Department for Environment, Food and Rural Affairs by Manchester Business School 
ISO 14040 (2006) Environmental management - life cycle assessment - principles and framework. Geneva

Jean-Michel Moutot, Ganaël Bascoul, Marketig et développement durable: Stratégie de la valeur étendure, Edition Dunod

Karli L, Horne R, Carre A (2010) PIQET: the design and development of an online 'streamlined' LCA tool for sustainable packaging design decision support. Int J Life Cycle Assess 15(6):608-620

Kim D, Thoma G, Nutter D, Milani F, Ulrich R, Norris G (2013) Life cycle assessment of cheese and whey production in the USA. Int J Life Cycle Assess 18(5):1019-1035

Peano L, De Schryver A, Humbert S, Loerincik Y, Gaillard G, Lansche J, Nemecek T (2012) The world food LCA database project: towards more accurate food datasets. In: Proceedings 2nd LCA conference, Nov 2012, France

Schenker U, Espinoza-Orias N, Popovic D (2014) EcodEX: a simplified ecodesign tool to improve the environmental performance of product development in the food industry. In: Proceedings of the 9th international conference on life cycle assessment in the agri-food sector, San Francisco, Oct 2014

Selerant Corporation (2015) EcodEX, Ecodesign Software. http://www.selerant.com/main/en-us/ solutions/ecodesign.aspx 